\title{
NECESSARY AND SUFFICIENT CONDITIONS FOR THE BOUNDEDNESS OF THE RIESZ POTENTIAL IN MODIFIED MORREY SPACES
}

\author{
VAgif S. Guliyev, JaVAnshir J. Hasanov and Yusuf Zeren
}

Abstract. We prove that the fractional maximal operator $M_{\alpha}$ and the Riesz potential operator $I_{\alpha}, 0<\alpha<n$ are bounded from the modified Morrey space $\widetilde{L}_{1, \lambda}\left(\mathbb{R}^{n}\right)$ to the weak modified Morrey space $W \widetilde{L}_{q, \lambda}\left(\mathbb{R}^{n}\right)$ if and only if, $\alpha / n \leqslant 1-1 / q \leqslant \alpha /(n-\lambda)$ and from $\widetilde{L}_{p, \lambda}\left(\mathbb{R}^{n}\right)$ to $\widetilde{L}_{q, \lambda}\left(\mathbb{R}^{n}\right)$ if and only if, $\alpha / n \leqslant 1 / p-1 / q \leqslant \alpha /(n-\lambda)$.

As applications, we establish the boundedness of some Schödinger type operators on modified Morrey spaces related to certain nonnegative potentials belonging to the reverse Hölder class. As an another application, we prove the boundedness of various operators on modified Morrey spaces which are estimated by Riesz potentials.

Mathematics subject classification (2010): Primary 42B20, 42B25, 42B35.

Keywords and phrases: Riesz potential, fractional maximal function, modified Morrey space, HardyLittlewood-Sobolev inequality, Schödinger type operator.

\section{REFERENCES}

[1] D.R. Adams, A note on Riesz potentials . Duke Math. 42 (1975), 765-778.

[2] D.R. ADAMS, Choquet integrals in potential theory, Publ. Mat. 42 (1998), 3-66.

[3] W. ARendt And A. F. M. TeR Elst, Gaussian estimates for second order elliptic operators with boundary conditions, J. Operator Theory 38 (1997), 87-130.

[4] P. Auscher And P. TChamitchian, Square root problem for divergence operators and related topics, Astérisque, 249, Soc. Math. France, 1998.

[5] V.I. BUREnKov, H.V. GuliYeV, Necessary and sufficient conditions for boundedness of the maximal operator in the local Morrey-type spaces, Studia Mathematica 163, 2 (2004), 157-176.

[6] V.I. BuREnKov, V.S. GuliYeV, Necessary and sufficient conditions for the boundedness of the Riesz operator in local Morrey-type spaces, Potential Analysis 30, 3 (2009), 211-249.

[7] L. Caffarelli, Elliptic second order equations, Rend. Sem. Mat. Fis. Milano 58 (1990), 253-284.

[8] F. Chiarenza, M. FrasCA, Morrey spaces and Hardy-Littlewood maximal function, Rend. Math. 7 (1987), 273-279.

[9] V.S. GuliYev, Sobolev's theorem for the anisotropic Riesz-Bessel potential in Morrey-Bessel spaces (Russian), Dokl. Akad. Nauk 367, 2 (1999), 155-156.

[10] V.S. GuliYev, On maximal function and fractional integral, associated with the Bessel differential operator, Mathematical Inequalities and Applications 6, 2 (2003), 317-330.

[11] V.S. GULIYEV AND J. HASANOV, Necessary and sufficient conditions for the boundedness of B-Riesz potential in the B-Morrey spaces, Journal of Mathematical Analysis and Applications 347, 1 (2008), $113-122$.

[12] X. T. Duong And L. X. YAn, On commutators of fractional integrals, Proc. Amer. Math. Soc. 132, 12 (2004), 3549-3557.

[13] G. Di Fazio, D.K. Palagachev and M.A. Ragusa, Global Morrey regularity of strong solutions to the Dirichlet problem for elliptic equations with discontinuous coefficients, J. Funct. Anal. 166 (1999), 179-196.

[14] C. Fefferman, The uncertainty principle, Bull. Amer. Math. Soc. 9 (1983), 129-206.

[15] C. Feffermann And E. Stein, Some maximal inequalities, Amer. J. Math. 93 (1971), 107-115. 
[16] A. Kufner, O. John And S. FuciK, Function Spaces, Noordhoff, Leyden, and Academia, Prague, 1977.

[17] K. Kurata, S. Sugano, A remark on estimates for uniformly elliptic operators on weighted $L_{p}$ spaces and Morrey spaces, Math. Nachr. 209 (2000), 137-150.

[18] H.Q. LI, Estimations $L_{p}$ des operateurs de Schrödinger sur les groupes nilpotents, J. Funct. Anal. 161 (1999), 152-218.

[19] YU LIU, The weighted estimates for the operators $V^{\alpha}\left(-\Delta_{G}+V\right)^{-\beta}$ and $V^{\alpha} \nabla_{G}\left(-\Delta_{G}+V\right)^{-\beta}$ on the stratified Lie group $\mathbb{G}$, J. Math. Anal. Appl. 349 (2009), 235-244.

[20] G.Z. LU, A Fefferman-Phong type inequality for degenerate vector fields and applications, Panamer. Math. J. 6 (1996), 37-57.

[21] A.L. MAZZUCATo, Besov-Morrey spaces: function space theory and applications to non-linear PDE, Trans. Amer. Math. Soc. 355 (2003), 1297-1364.

[22] A. McIntosh, Operators which have an $H_{1}$-calculusin, in Proc. Centre Math. Analysis, Vol. 14, Miniconference on Operator Theory and Partial Differential Equations, A. N. U., Canberra, 1986, 210231.

[23] B. Muckenhoupt And R. Wheeden, Weighted norm inequalities for fractional integrals, Trans. Amer. Math. Soc. 192 (1974), 261-274.

[24] C.B. Morrey, On the solutions of quasi-linear elliptic partial differential equations, Trans. Amer. Math. Soc. 43 (1938), 126-166.

[25] C. Perez, Two weighted norm inequalities for Riesz potentials and uniform $L_{p}$-weighted Sobolev inequalities, Indiana Univ. Math. J. 39 (1990), 31-44.

[26] B. Rubin, Fractional integrals and potentials, Pitman Monographs and Surveys in Pure and Applied Mathematics, 82. Longman, Harlow, 1996. xiv+409 pp.

[27] A. RUiz AND L. Vega, Unique continuation for Schrödinger operators with potential in Morrey spaces, Publ. Mat. 35 (1991), 291-298.

[28] A. Ruiz And L. Vega, On local regularity of Schrödinger equations, Int. Math. Res. Notices 1993, 1 (1993), 13-27.

[29] S.G. Samko, A.A. Kilbas, O.I. MaricheV, Fractional integrals and derivatives. Theory and applications. Gordon and Breach Science Publishers, Yverdon, 1993. xxxvi+976 pp.

[30] Z.W. SHEN, $L_{p}$ estimates for Schrödinger operators with certain potentials, Ann. Inst. Fourier (Grenoble) 45 (1995), 513-546.

[31] Z. SHEN, The periodic Schrödinger operators with potentials in the Morrey class, J. Funct. Anal. 193 (2002), 314-345.

[32] Z. SHEN, Boundary value problems in Morrey spaces for elliptic systems on Lipschitz domains, Amer. J. Math. 125 (2003), 1079-1115.

[33] B. Simon, Maximal and minimal Schrödinger forms, J. Op. Theory 1 (1979), 37-47.

[34] E.M. SteIn, Singular integrals and differentiability properties of functions, Princeton Univ. Press, Princeton, NJ, 1970.

[35] E.M. SteIn AND G. WeIss, Introduction to Fourier analysis on Euclidean spaces, Princeton Univ. Press, Princeton, NJ, 1971.

[36] S. SugAno, Estimates for the operators $V^{\alpha}(-\Delta+V)^{-\beta}$ and $V^{\alpha} \nabla(-\Delta+V)^{-\beta}$ with certain nonnegative potentials $V$, Tokyo J. Math. 21 (1998), 441-452.

[37] M.E. TAYLOR, Analysis on Morrey spaces and applications to Navier-Stokes and other evolution equations, Comm. Partial Differential Equations 17 (1992), 1407-1456.

[38] J.P. ZHONG, Harmonic analysis for some Schrödinger type operators, $\mathrm{PhD}$ thesis, Princeton University, 1993. 